\title{
Atomic data for M1 and E2 emission lines in the potassium isoelectronic sequence ${ }^{\star}$
}

\author{
E. Charro, Z. Curiel, and I. Martín \\ Departamento de Química Física, Universidad de Valladolid, 47011 Valladolid, Spain
}

Received 8 October 2001 / Accepted 8 February 2002

\begin{abstract}
Relativistic Quantum Defect Orbital (RQDO) calculations of transition probabilities for E2 and M1 forbidden transition in the potassium sequence have been performed. Intensities for the higher ions are reported, to our knowledge, for the first time, as they are potentially important for the study of the plasma in astrophysical objects and fusion devices.
\end{abstract}

Key words. atomic data - atomic processes

\section{Introduction}

Theoretical studies of atomic transition probabilities have mostly concentrated on dipole-allowed (E1) transitions, as these are usually responsible for strong lines in atomic spectra. However, it is now realized that under conditions which prevail in many astrophysical and low-density laboratory tokamak plasmas, collisional de-excitation of metastable states is rather slow, leading to buildup of a population of metastable levels. There, forbidden transitions, i.e., electric quadrupole (E2) and magnetic dipole (M1) transitions, gain in intensity and can be used to infer information about plasma temperature and dynamics. A selection of forbidden lines arising through electric quadrupole (E2) and magnetic dipole (M1) transitions in many atoms and ions can be used as a basis for accurate electron density and/or temperature diagostics in various astronomical objects or in laboratory tokamak plasmas (Seaton 1968; Gabriel \& Jordan 1969; Osterbrock 1974, 1989; Suckewer \& Hinnov 1979; Hinnov \& Suckewer 1980; Suckewer et al. 1980; Edlén 1984). Although most of these lines are weak, they can produce systematic errors in analyses if their presence is ignored (Adelman \& Gulliver 1999).

Radiative transitions between energy levels within the ground configuration of atoms and atomic ions are strictly forbidden to proceed via the emission of

\footnotetext{
Send offprint requests to: E. Charro,

e-mail: Imnieto@wamba.cpd.uva.es

* Tables 1 to 5 are also available in electronic form at the CDS via anonymous $\mathrm{ftp}$ to cdsarc.u-strasbg.fr (130.79.128.5) or via

http://cdsweb.u-strasbg.fr/cgi-bin/qcat?J/A+A/387/1146
}

electric dipole (E1) radiation because, for levels within the ground configuration, the parity of the upper and lower levels does not differ. The lowest-order, nonzero, radiative moments by which such metastable levels radiatively decay correspond to magnetic dipole (M1) and electric quadrupole (E2) transitions. In low-charge atomic ions, the M1 and E2 transition probabilities (A values) are typically several orders of magnitude smaller than those for E1 transitions with a similar energy level separation. As a consequence, radiative lifetimes of metastable levels in these ions range from hundreds of microseconds to many seconds (Calamai et al. 2000).

Experimentally, spectra of some K-like ions have been analysed. For instance, spectra of $\mathrm{Mo}^{23+}$ were identified in laboratory plasmas (Kaufman et al. 1989). The electron temperature and density dependence of E1 and E2 lines in the spectra of potassium-like molybdenum has been analysed by Fournier et al. (1996), given its role in magnetically confined fusion experiments. An analysis of the spectrum of Ni X (Wang et al. 1999) and Mn VII (Wang et al. 1997) has been reported following observations involving collision spectroscopy. However, in view of the paucity of experimental data on fine-structure splittings of highly ionized atoms, some theoretical effort has been devoted to prediction in ions with high nuclear charge $Z$ in order to identify such lines in plasmas.

A number of calculations have been done on the ions isoelectronic with $\mathrm{K}$, most of them corresponding to allowed transitions. Both the non-relativistic (QDO) and the relativistic formulation (RQDO) of the quantum defect orbital method have been applied to the study of E1 transitions for a group of atoms of this sequence (Martín et al. 1991, 2000a). For forbidden transitions, the data is 
more scarce. Theoretical E2 and M1 transition probabilities along the potassium sequence up to $Z=42$ have been published by Ali \& Kim (1988); Biémont (1990) has reported values for ten ions from $Z=48$ onwards; calculations for ions between $Z=22$ and $Z=47$ have been performed by Biémont \& Hansen (1990).

However, to our knowledge, data on E2 and M1 transition probabilities for the whole sequence have not been reported. We have thus considered that there is room for new calculations of transition probability data for these two kinds of forbidden lines in the potassium sequence.

Over the last decade, we have applied the RQDO method (Martín \& Karwowski 1991; Karwowski \& Martín 1991; Martín et al. 2000b) to the calculation of oscillator strengths and photoionization cross sections of several isoelectronic sequences (see, e.g. Martín et al. 1991, 1993, 1994, 1995, 2000a; Charro \& Martín 1998; Charro et al. 1997, 2000). The RQDO formalism, is a simple but reliable analytical method based on a model Hamiltonian. It has the great advantage that the computational effort does not increase as the atomic system dealt with becomes heavier. The convenience of employing exactly solvable model potentials for calculating atomic transition probabilities manifests itself not only from a practical point of view but also because of the involved physical implications, when they are capable of achieving a good balance between computational effort and accuracy of results.

In the present work, we have undertaken the RQDO study of the intensities of several electric quadrupole (E2) and magnetic dipole (M1) emission lines, involving $3 \mathrm{~d}_{3 / 2}$, $3 \mathrm{~d}_{5 / 2}$ and $4 \mathrm{~s}_{1 / 2}$ levels, in a number of K-like ions ranging from Mn VII $(Z=25)$ to $\operatorname{Hg} \operatorname{LXII}(Z=80)$. As some of the ions studied are heavy and in a high degree of ionization, the relativistic contributions to the wavefunctions and energies of the levels involved in the present transitions may not be negligible. The $A$-values have been calculated on an individual basis, rather than from the application of the $L S$-coupling rules within multiplets. We find the direct calculation of fine-structure line strengths to be interesting from a spectroscopic point of view, given their usefulness in spectral analysis in astrophysics and fusion plasma research. A general good agreement has been found between our data and other results.

\section{Computational procedure}

The Relativistic Quantum Defect Orbital (RQDO) method has been described in detail in previous papers (Karwowski \& Martín 1991; Martín et al. 1993). Therefore, we shall only briefly summarise its most fundamental aspects.

The relativistic quantum defect orbitals are determined by solving analytically the quasi-relativistic scalar second-order Dirac-like equation, obtained after decoupling the radial, two-component Dirac equation, through a non-unitary transformation.

$\left[-\frac{\mathrm{d}^{2}}{\mathrm{~d} r^{2}}+\frac{\Lambda(\Lambda+1)}{r^{2}}-\frac{2 Z_{\text {net }}^{\prime}}{r}\right] \psi_{k}^{\mathrm{RD}}=2 e^{\mathrm{RD}}{\psi_{k}}^{\mathrm{RD}}$, with

$Z_{\text {net }}^{\prime}=Z_{\text {net }}\left(1+\alpha^{2} E^{x}\right)$

$e^{\mathrm{RD}}=-\frac{\left(Z_{\mathrm{net}}^{\prime}\right)^{2}}{2\left(\eta-\delta^{\prime}\right)^{2}}=E^{x} \frac{\left(1+\alpha^{2} E^{x} / 2\right)}{\left(1+\alpha^{2} E^{x}\right)^{2}}$

and,

$\Lambda=s-1-\delta^{\prime}+c$,

when $j=l-1 / 2$, or

$\Lambda=-s-\delta^{\prime}+c$,

when $j=l+1 / 2$, with

$s=k\left(1-\alpha^{2} Z^{2} / k^{2}\right)^{1 / 2}$

and

$k=\epsilon(j+1 / 2)$

being $\epsilon= \pm 1$.

Here $n$ and $l$ are the principal and orbital angular momentum quantum numbers; and $\eta$ is the relativistic principal quantum number, related to $n$ as follows

$\eta=n-|k|+|s|$

$\delta^{\prime}$ is the relativistic quantum defect, $c$ is an integer chosen to ensure the normalizability of the wavefunction and its correct nodal structure; $Z_{\text {net }}^{\prime}$ is the scaled nuclear charge acting on the valence electrons at large radial distances; $E^{x}$ is the experimentally measured energy, and $\alpha$ is the fine structure constant. Atomic units are used throughout. Since the effective Hamiltonian in Eq. (2.1) includes a screening term, the quantum defect orbitals are approximately valid in the core region of space. Core polarization effects are implicitly included in the calculations given that they are accounted for in the $\Lambda$ parameter of the model Hamiltonian. On the other hand, because of the one-electron nature of the RQDO formalism, it can be expected to perform better in highly excited states, where the active electron interacts less with the core and other valence electrons, than in low-lying energy states. The relativistic quantum defect orbitals lead to closed-form analytical expresions for the transition integrals. This allows us to calculate transition probabilities and oscillator strengths without any convergence problems.

\section{E2 and M1 forbidden transitions}

Our methodology supplies one-electron radial wavefunctions, characterized by the $n, l$ and $j$ quantum numbers (Martín \& Karwowski 1991), that we employ in the transition matrix elements for the initial and final states of the active electron. These correspond to levels of a given $L, S$ and $J$ symmetry in many-electron atoms. Thus, within the $L S$ coupling, $L=l$ as the alkali-like systems have only one optical electron. The electric quadrupole line strength for a transition between two states within 
the $L S J$-coupling, which is the coupling scheme followed throughout in this work, is given by the equation

$$
\begin{aligned}
& S_{\mathrm{E} 2}\left(n l j, n^{\prime} l^{\prime} j^{\prime}\right)=\frac{2}{3}(2 J+1)\left(2 J^{\prime}+1\right) W\left(S J L^{\prime} 2, L J^{\prime}\right)^{2} \\
& R_{\mathrm{mult}}^{2}\left(\alpha L, \alpha^{\prime} L^{\prime}\right) \times\left\langle L\left\|C^{(2)}\right\| L^{\prime}\right\rangle^{2}\left|\left\langle R_{n l j}|Q(r)| R_{n^{\prime} l^{\prime} j^{\prime}}\right\rangle\right|^{2}
\end{aligned}
$$

where $Q(r)$ is the quadrupole transition operator, $r^{2}$, $R_{\text {mult }}$ is the multiplet factor, $W\left(S J L^{\prime} 2, L J^{\prime}\right)$ are the Racah coefficients which can be described in terms of $6 j$ symbols

$$
W\left(S J L^{\prime} 2, L J^{\prime}\right)=(-1)^{S+J+L^{\prime}+2}\left\{\begin{array}{lll}
S & J & L \\
2 & L^{\prime} & J^{\prime}
\end{array}\right\}
$$

and $\left\langle L\left\|C^{(2)}\right\| L^{\prime}\right\rangle$ is the pertinent reduced matrix element, which can be evaluated using $3 j$-symbols by the following expresion

$$
\left\langle L\left\|C^{(2)}\right\| L^{\prime}\right\rangle=\left[L, L^{\prime}\right]\left(\begin{array}{ccc}
L & 2 & L^{\prime} \\
0 & 0 & 0
\end{array}\right) .
$$

The relationships between the oscillator strength $f_{\mathrm{E} 2}$ (dimensionless), the transition probability $A_{\mathrm{E} 2}\left(\right.$ in $\left.\mathrm{s}^{-1}\right)$ and the line strength $S_{\mathrm{E} 2}$ (in atomic units, $e^{2} a_{\mathrm{o}}^{4}$ ), for E2 transitions are given by (Sobelman 1972)

$$
A_{\mathrm{E} 2}=\frac{8 \pi^{2} \hbar \alpha}{m \lambda^{2}} \frac{g}{g^{\prime}} f_{\mathrm{E} 2}=6.6703 \times 10^{15} \frac{g f_{\mathrm{E} 2}}{g^{\prime} \lambda^{2}}
$$

and by

$$
A_{\mathrm{E} 2}=\left(32 \pi^{5} \alpha c a_{\mathrm{o}}^{4} / 15 \lambda^{5}\right) \frac{S_{\mathrm{E} 2}}{g^{\prime}}=\left(1.11995 \times 10^{18} / \lambda^{5}\right) \frac{S_{\mathrm{E} 2}}{g^{\prime}},
$$

respectively, where $\lambda$ is the transition wavelength (in $\AA$ ), $g$ and $g^{\prime}$ are the degeneracy of the lower and upper states, and $\alpha$ is the fine-structure constant.

The formulae for the probability of magnetic dipole (M1) transitions do not contain radial integrals. Thus, the line strength, $S_{\mathrm{E} 2}$, which is independent of the frequency of the transition, can be expressed as

$$
\begin{aligned}
S_{\mathrm{M} 1}(n l j, n l j-1) & =\frac{1}{4 j}(l+1 / 2+j+1)(l+1 / 2-j+1) \\
& \times(1 / 2+j-l)(j+l-1 / 2) .
\end{aligned}
$$

The transition probability $A_{\mathrm{E} 2}\left(\right.$ in $\mathrm{s}^{-1}$ ) is related to the line strength $S_{\mathrm{M} 1}$, in Bohr magneton units $\mu_{\mathrm{B}}{ }^{2}$, by the expression

$A_{\mathrm{E} 2}=\left(2.697 \times 10^{13} / \lambda^{3}\right) \frac{S_{\mathrm{E} 2}}{g^{\prime}}$.

The relationship between the oscillator strength $f_{\mathrm{M} 1}$ (dimensionless) and the transition probability $A_{\mathrm{M} 1}\left(\right.$ in s $\left.^{-1}\right)$ is given by Eq. (3.4), which is generally valid for E1, E2 and M1 transitions.

\section{Energy data}

\subsection{Energy levels}

The distribution of the energy levels along the potassium isoelectronic sequence is characterized by some changes in the possition of the $4 \mathrm{~s}^{2} \mathrm{~S}_{1 / 2}, 3 \mathrm{~d}{ }^{2} \mathrm{D}_{3 / 2}$ and $3 \mathrm{~d}{ }^{2} \mathrm{D}_{5 / 2}$ states. The ground state in K I and Ca II corresponds to the $4 \mathrm{~s}^{2} \mathrm{~S}_{1 / 2}$ level. However, for the rest of the ions belonging to this sequence, the $3 \mathrm{~d}^{2} \mathrm{D}_{3 / 2}$ level has to be considered as the ground state. The RQDO calculations have been performed on the emission processes (via electric quadrupole and magnetic dipole mechanism) experienced by the atomic systems, and involving, in all cases, a decay to the ground state, $3 \mathrm{~d}^{2} \mathrm{D}_{3 / 2}$. Given the semiempirical character of our methodology, experimental energy data are required. For the $4 \mathrm{~s}^{2} \mathrm{~S}_{1 / 2}, 3 \mathrm{~d}^{2} \mathrm{D}_{3 / 2}$ and $3 \mathrm{~d}^{2} \mathrm{D}_{5 / 2}$ levels, we have employed the values provided by Ali \& Kim (1988), which have been taken from different sources, most of them from the compilation by Sugar \& Corliss (1985). These data are available up to Mo XXIV. For the ions for which no data were found, extrapolated or interpolated energy values have been used. In order to do this, using the experimental data we tested several fitting polynomial expressions with dependence on the nuclear charge, $Z$. The ones obtained for the $3 \mathrm{~d}^{2} \mathrm{D}_{5 / 2}$ and $4 \mathrm{~s}^{2} \mathrm{~S}_{1 / 2}$ levels with the best correlation factor (0.9999) where the following

$E_{3 \mathrm{~d}_{5 / 2}}=3.35 Z^{3}-210 Z^{2}+4666 Z-36144$

$E_{4 \mathrm{~s}_{1 / 2}}=-14.42 Z^{3}+6677 Z^{2}-210000 Z+1621 \times 10^{3}$

where $E$ is in $\mathrm{cm}^{-1}$.

As we advance in the sequence, a $Z$-value will be reached for which the spin-orbit coupling scheme can no longer be expected to be pure $L S$ but an intermediate one between that and jj coupling. However, we have not changed, however, the formulation of either the radial functions or the angular factors employed in the calculations at any point in the sequence. We expect that the use of empirical energy data will, at least to some extent, account for intermediate coupling, as it does at all values of $Z$, for some of the configuration interaction.

\subsection{Ionization energy}

Other input data also needed in the RQDO calculations are the ionization energies (I.E.) of the atomic systems. For K I to Ni X we have adopted the IE supplied by Sugar \& Corliss (1985), and from Cu XI up to Se XVI the ones reported by Kelly (1987). For the remaining ions, we have employed an extrapolation formula obtained by fitting the previous ionization energies in $\mathrm{cm}^{-1}$ (that is, the $I . E$. of the ions between $Z=21$ and $Z=34$ ). This formula is the following

I.E. $=2838000-393000 Z+12742 Z^{2}$

where, again, $Z$ is the atomic number. The correlation factor is 0.9999 . 
Table 1. Transition probabilities for $3 \mathrm{~d}_{3 / 2} \leftarrow 4 \mathrm{~s}_{1 / 2}$ E2 spectral line.

\begin{tabular}{lllll}
\hline \hline & & This & Ali- & \\
ION & $Z$ & work $^{a}$ & Kim $^{b}$ & Biémont $^{c}$ \\
\hline Mn VII & 25 & $1.44(+5)$ & $1.22(+5)$ & - \\
Fe VIII & 26 & $3.46(+5)$ & $3.09(+5)$ & - \\
Co IX & 27 & $7.19(+5)$ & $6.69(+5)$ & - \\
Ni X & 28 & $1.35(+6)$ & $1.30(+6)$ & - \\
Cu XI & 29 & $2.34(+6)$ & $2.33(+6)$ & - \\
Zn XII & 30 & $3.84(+6)$ & $3.93(+6)$ & - \\
Ga XIII & 31 & $6.00(+6)$ & $6.32(+6)$ & - \\
Ge XIV & 32 & $9.05(+6)$ & $9.72(+6)$ & - \\
As XV & 33 & $1.32(+7)$ & $1.45(+7)$ & - \\
Se XVI & 34 & $1.87(+7)$ & $2.09(+7)$ & - \\
Br XVII & 35 & $2.63(+7)$ & $2.95(+7)$ & - \\
Kr XVIII & 36 & $3.59(+7)$ & $4.07(+7)$ & - \\
Rb XIX & 37 & $4.81(+7)$ & $5.52(+7)$ & - \\
Sr XX & 38 & $6.35(+7)$ & $7.36(+7)$ & - \\
Y XXI & 39 & $8.27(+7)$ & $9.66(+7)$ & - \\
Zr XXII & 40 & $1.06(+8)$ & $1.25(+8)$ & - \\
Nb XXIII & 41 & $1.35(+8)$ & $1.60(+8)$ & - \\
Mo XXIV & 42 & $1.70(+8)$ & $2.03(+8)$ & - \\
Tc XXV & 43 & $2.13(+8)$ & - & - \\
Ru XXVI & 44 & $2.63(+8)$ & - & - \\
Rh XXVII & 45 & $3.23(+8)$ & - & - \\
Pd XXVIII & 46 & $3.94(+8)$ & - & - \\
Ag XXIX & 47 & $4.77(+8)$ & - & - \\
Cd XXX & 48 & $5.74(+8)$ & - & $7.53(+8)$ \\
In XXXI & 49 & $6.86(+8)$ & - & $9.03(+8)$ \\
Sn XXXII & 50 & $8.17(+8)$ & - & $1.08(+9)$ \\
Sb XXXIII & 51 & $9.67(+8)$ & - & $1.28(+9)$ \\
Te XXXIV & 52 & $1.14(+9)$ & - & $1.51(+9)$ \\
I XXXV & 53 & $1.34(+9)$ & - & $1.77(+9)$ \\
Xe XXXVI & 54 & $1.56(+9)$ & - & $2.71(+9)$ \\
Cs XXXVII & 55 & $1.82(+9)$ & - & - \\
Ba XXXVIII & 56 & $2.10(+9)$ & - & - \\
La XXXIX & 57 & $2.43(+9)$ & - & - \\
Ce XL & 58 & $2.80(+9)$ & - & - \\
\hline & & & & - \\
\hline
\end{tabular}

In this and the remaining tables, $A(B)$ denotes $A \cdot 10^{(B)}$.

${ }^{a}$ Relativistic Quantum Defect Orbital Method, this work.

${ }^{b}$ MCDF (Ali \& Kim 1988).

${ }^{c}$ MCDF (Biémont 1990).

\section{Results and analysis}

For whole the ions belonging to this sequence and for both initial and final states involved in the E2 and M1 transitions studied here, the same value of $c$, mentioned in Eqs. (2.4) and (2.5), has been chosen. The possible values of the parameter $c$ are determined by the following expression:

$c \leq n-l-1$.
Table 2. Transition probabilities for $3 \mathrm{~d}_{5 / 2} \leftarrow 4 \mathrm{~s}_{1 / 2}$ E2 spectral line.

\begin{tabular}{|c|c|c|c|c|}
\hline ION & $Z$ & $\begin{array}{l}\text { This } \\
\text { work }^{a}\end{array}$ & $\begin{array}{l}\text { Ali- } \\
\text { Kim }^{b}\end{array}$ & Biémont $^{c}$ \\
\hline Mn VII & 25 & $2.13(+5)$ & $1.82(+5)$ & - \\
\hline Fe VIII & 26 & $5.12(+5)$ & $4.59(+5)$ & - \\
\hline Co IX & 27 & $1.06(+6)$ & $9.94(+5)$ & - \\
\hline Ni X & 28 & $1.99(+6)$ & $1.93(+6)$ & - \\
\hline $\mathrm{Cu} \mathrm{XI}$ & 29 & $3.46(+6)$ & $3.47(+6)$ & - \\
\hline Zn XII & 30 & $5.68(+6)$ & $5.84(+6)$ & - \\
\hline Ga XIII & 31 & $8.87(+6)$ & $9.38(+6)$ & - \\
\hline Ge XIV & 32 & $1.34(+7)$ & $1.44(+7)$ & - \\
\hline As XV & 33 & $1.94(+7)$ & $2.15(+7)$ & - \\
\hline Se XVI & 34 & $2.76(+7)$ & $3.11(+7)$ & - \\
\hline Br XVII & 35 & $3.87(+7)$ & $4.38(+7)$ & - \\
\hline Kr XVIII & 36 & $5.28(+7)$ & $6.04(+7)$ & - \\
\hline Rb XIX & 37 & $7.07(+7)$ & $8.18(+7)$ & - \\
\hline Sr XX & 38 & $9.33(+7)$ & $1.09(+8)$ & - \\
\hline Y XXI & 39 & $1.21(+8)$ & $1.43(+8)$ & - \\
\hline Zr XXII & 40 & $1.56(+8)$ & $1.86(+8)$ & - \\
\hline Nb XXIII & 41 & $1.98(+8)$ & $2.37(+8)$ & - \\
\hline Mo XXIV & 42 & $2.50(+8)$ & $3.01(+8)$ & - \\
\hline Tc XXV & 43 & $3.11(+8)$ & - & - \\
\hline Ru XXVI & 44 & $3.84(+8)$ & - & - \\
\hline Rh XXVII & 45 & $4.72(+8)$ & - & - \\
\hline Pd XXVIII & 46 & $5.74(+8)$ & - & - \\
\hline Ag XXIX & 47 & $6.95(+8)$ & - & - \\
\hline Cd XXX & 48 & $8.35(+8)$ & - & $1.13(+9)$ \\
\hline In XXXI & 49 & $9.98(+8)$ & - & $1.36(+9)$ \\
\hline Sn XXXII & 50 & $1.19(+9)$ & - & $1.62(+9)$ \\
\hline Sb XXXIII & 51 & $1.40(+9)$ & - & $1.93(+9)$ \\
\hline Te XXXIV & 52 & $1.65(+9)$ & - & $2.27(+9)$ \\
\hline I XXXV & 53 & $1.94(+9)$ & - & $2.67(+9)$ \\
\hline Xe XXXVI & 54 & $2.26(+9)$ & - & $3.12(+9)$ \\
\hline Cs XXXVII & 55 & $2.62(+9)$ & - & $3.64(+9)$ \\
\hline Ba XXXVIII & 56 & $3.03(+9)$ & - & $4.22(+9)$ \\
\hline La XXXIX & 57 & $3.44(+9)$ & - & $4.87(+9)$ \\
\hline Ce XL & 58 & $4.02(+9)$ & - & $5.61(+9)$ \\
\hline
\end{tabular}

See footnotes in Table 1.

The integer $c$ has been taken equal to zero for both $4 \mathrm{~s}$ and $3 \mathrm{~d}$ states. This leads to RQDO wavefunctions that possess the same number of radial nodes as a hydrogenic function.

RQDO values of transition probabilities for E2 and M1 emission lines for fifty five ions isoelectronic with potassium are reported. As already mentioned, we have used the experimental transition energies where they are available, and fitted values for the energies calculated according to the expressions given in the previous section. Only for M1 transitions, the $\lambda$ estimated from our fitted expressions 
Table 3. E2 Transition probabilities for $3 \mathrm{~d}_{3 / 2} \leftarrow 3 \mathrm{~d}_{5 / 2}$ spectral line.

\begin{tabular}{|c|c|c|c|c|c|}
\hline ION & $Z$ & $\begin{array}{l}\text { This } \\
\text { work }^{a}\end{array}$ & $\begin{array}{l}\text { Ali- } \\
\text { Kim }^{b}\end{array}$ & Biémont $^{c}$ & $\begin{array}{l}\text { Biémont- } \\
\text { Hansen }^{d}\end{array}$ \\
\hline Mn VII & 25 & $2.22(-8)$ & $2.10(-8)$ & - & $2.21(-8)$ \\
\hline Fe VIII & 26 & $7.04(-8)$ & $7.04(-8)$ & - & $7.09(-8)$ \\
\hline Co IX & 27 & $2.05(-7)$ & $2.14(-7)$ & - & $2.16(-7)$ \\
\hline Ni X & 28 & $5.34(-7)$ & $5.80(-7)$ & - & $5.84(-7)$ \\
\hline $\mathrm{Cu} \mathrm{XI}$ & 29 & $1.34(-6)$ & $1.41(-6)$ & - & $1.51(-6)$ \\
\hline Zn XII & 30 & $2.74(-6)$ & $3.41(-6)$ & - & $3.64(-6)$ \\
\hline Ga XIII & 31 & $6.74(-6)$ & $7.85(-6)$ & - & $8.34(-6)$ \\
\hline Ge XIV & 32 & $1.45(-5)$ & $1.73(-5)$ & - & $1.83(-5)$ \\
\hline As XV & 33 & $3.04(-5)$ & $3.69(-5)$ & - & $3.84(-5)$ \\
\hline Se XVI & 34 & $6.05(-5)$ & $7.41(-5)$ & - & $7.80(-5)$ \\
\hline Br XVII & 35 & $1.17(-4)$ & $1.46(-4)$ & - & $1.53(-4)$ \\
\hline Kr XVIII & 36 & $2.21(-4)$ & $2.79(-4)$ & - & $2.93(-4)$ \\
\hline Rb XIX & 37 & $4.08(-4)$ & $5.20(-4)$ & - & $5.44(-4)$ \\
\hline Sr XX & 38 & $7.34(-4)$ & $9.44(-4)$ & - & $9.87(-4)$ \\
\hline Y XXI & 39 & $1.29(-3)$ & $1.68(-3)$ & - & $1.75(-3)$ \\
\hline Zr XXII & 40 & $2.31(-3)$ & $2.91(-3)$ & - & $3.04(-3)$ \\
\hline Nb XXIII & 41 & $3.78(-3)$ & $4.97(-3)$ & - & $5.19(-3)$ \\
\hline Mo XXIV & 42 & $6.49(-3)$ & $8.33(-3)$ & - & $8.68(-3)$ \\
\hline Tc XXV & 43 & $1.10(-2)$ & - & - & $1.43(-2)$ \\
\hline Ru XXVI & 44 & $1.75(-2)$ & - & - & $2.32(-2)$ \\
\hline Rh XXVII & 45 & $2.74(-2)$ & - & - & $3.70(-2)$ \\
\hline Pd XXVIII & 46 & $4.21(-2)$ & - & - & $5.84(-2)$ \\
\hline Ag XXIX & 47 & $6.39(-2)$ & - & - & $9.03(-2)$ \\
\hline Cd XXX & 48 & $9.57(-2)$ & - & $1.31(-1)$ & - \\
\hline In XXXI & 49 & $1.41(-1)$ & - & $2.00(-1)$ & - \\
\hline Sn XXXII & 50 & $2.07(-1)$ & - & $3.01(-1)$ & - \\
\hline Sb XXXIII & 51 & $2.98(-1)$ & - & $4.48(-1)$ & - \\
\hline Te XXXIV & 52 & $4.26(-1)$ & - & $6.60(-1)$ & - \\
\hline I XXXV & 53 & $6.02(-1)$ & - & $9.65(-1)$ & - \\
\hline Xe XXXVI & 54 & $8.42(-1)$ & - & $1.40(+0)$ & - \\
\hline Cs XXXVII & 55 & $1.17(+0)$ & - & $2.01(+0)$ & - \\
\hline Ba XXXVIII & 56 & $1.61(+0)$ & - & $2.86(+0)$ & - \\
\hline La XXXIX & 57 & $2.19(+0)$ & - & $4.04(+0)$ & - \\
\hline Ce XL & 58 & $2.96(+0)$ & - & $5.67(+0)$ & - \\
\hline
\end{tabular}

${ }^{a}{ }^{c}$ See footnotes in Table 1.

${ }^{d}$ Relativistic Hartree-Fock approach (Biémont \& Hansen 1989).

have been employed for all the ions. The line strength, $S_{\mathrm{M} 1}$, is a constant value for all the ions, equal to $2.4 \mu_{\mathrm{B}}{ }^{2}$.

The calculated electric quadrupole inter- and intraconfiguration transition probabilities between doublets of the K-like ions between Mn VII $(Z=25)$ and Ce XL $(Z=$ 58) are displayed in Tables $1-3$. Tables 1 and 2 collect the interconfiguration $3 \mathrm{~d}-4 \mathrm{~s}$ arrays, with the $3 \mathrm{~d}^{2} \mathrm{D}_{3 / 2} \leftarrow$ $4 \mathrm{~s}^{2} \mathrm{~S}_{1 / 2}$ and $3 \mathrm{~d}^{2} \mathrm{D}_{5 / 2} \leftarrow 4 \mathrm{~s}^{2} \mathrm{~S}_{1 / 2}$ transitions, respectively. The $A$-values for the $3 \mathrm{~d}^{2} \mathrm{D}_{3 / 2} \leftarrow 3 \mathrm{~d}^{2} \mathrm{D}_{5 / 2}$ line, corresponding to the $\mathrm{E} 2$ intraconfiguration transition, are
Table 4. M1 Transition probabilities for $3 \mathrm{~d}_{3 / 2} \leftarrow 3 \mathrm{~d}_{5 / 2}$ spectral line.

\begin{tabular}{llllll}
\hline \hline & & This & Ali- & & Biémont- \\
ION & $Z$ & work $^{a}$ & Kim $^{b}$ & Biémont $^{c}$ & Hansen $^{d}$ \\
\hline Mn VII & 25 & $4.42(-2)$ & $2.58(-2)$ & - & $2.67(-2)$ \\
Fe VIII & 26 & $9.87(-2)$ & $6.67(-2)$ & - & $6.70(-2)$ \\
Co IX & 27 & $2.09(-1)$ & $1.59(-1)$ & - & $1.60(-1)$ \\
Ni X & 28 & $4.25(-1)$ & $3.46(-1)$ & - & $3.48(-1)$ \\
Cu XI & 29 & $8.36(-1)$ & $6.95(-1)$ & - & $7.25(-1)$ \\
Zn XII & 30 & $1.59(+0)$ & $1.38(+0)$ & - & $1.43(+0)$ \\
Ga XIII & 31 & $2.95(+0)$ & $2.62(+0)$ & - & $2.72(+0)$ \\
Ge XIV & 32 & $5.32(+0)$ & $4.81(+0)$ & - & $4.98(+0)$ \\
As XV & 33 & $9.35(+0)$ & $8.54(+0)$ & - & $8.83(+0)$ \\
Se XVI & 34 & $1.60(+1)$ & $1.47(+1)$ & - & $1.52(+1)$ \\
Br XVII & 35 & $2.68(+1)$ & $2.48(+1)$ & - & $2.56(+1)$ \\
Kr XVIII & 36 & $4.39(+1)$ & $4.07(+1)$ & - & $4.36(+1)$ \\
Rb XIX & 37 & $7.05(+1)$ & $6.54(+1)$ & - & $6.74(+1)$ \\
Sr XX & 38 & $1.11(+2)$ & $1.03(+2)$ & - & $1.06(+2)$ \\
Y XXI & 39 & $1.71(+2)$ & $1.60(+2)$ & - & $1.65(+2)$ \\
Zr XXII & 40 & $2.60(+2)$ & $2.44(+2)$ & - & $2.51(+2)$ \\
Nb XXIII & 41 & $3.89(+2)$ & $3.67(+2)$ & - & $3.77(+2)$ \\
Mo XXIV & 42 & $5.73(+2)$ & $5.44(+2)$ & - & $5.59(+2)$ \\
Tc XXV & 43 & $8.31(+2)$ & - & - & $8.17(+2)$ \\
Ru XXVI & 44 & $1.20(+3)$ & - & - & $1.18(+3)$ \\
Rh XXVII & 45 & $1.68(+3)$ & - & - & $1.69(+3)$ \\
Pd XXVIII & 46 & $2.36(+3)$ & - & - & $2.38(+3)$ \\
Ag XXIX & 47 & $3.26(+3)$ & - & - & $3.32(+3)$ \\
Cd XXX & 48 & $4.48(+3)$ & - & $4.44(+3)$ & - \\
In XXXI & 49 & $6.09(+3)$ & - & $6.10(+3)$ & - \\
Sn XXXII & 50 & $8.11(+3)$ & - & $8.32(+3)$ & - \\
Sb XXXIII & 51 & $1.08(+4)$ & - & $1.13(+4)$ & - \\
Te XXXIV & 52 & $1.42(+4)$ & - & $1.51(+4)$ & - \\
I XXXV & 53 & $1.87(+4)$ & - & $2.01(+4)$ & - \\
Xe XXXVI & 54 & $2.42(+4)$ & - & $2.67(+4)$ & - \\
Cs XXXVII & 55 & $3.13(+4)$ & - & $3.51(+4)$ & - \\
Ba XXXVIIII & 56 & $4.00(+4)$ & - & $4.59(+4)$ & - \\
La XXXIX & 57 & $5.10(+4)$ & - & $5.97(+4)$ & - \\
Ce XL & 58 & $6.48(+4)$ & - & $7.72(+4)$ & - \\
\hline & & & & & \\
\hline
\end{tabular}

See footnotes in Table 3.

collected in Table 3. In Table 4, the transition probabilities for the $3 \mathrm{~d}^{2} \mathrm{D}_{3 / 2} \leftarrow 3 \mathrm{~d}^{2} \mathrm{D}_{5 / 2} \mathrm{M} 1$ transition are reported.

In these tables, the most recent comparative data found in the literature, all of which have been obtained with theoretical calculations, have been included. No experimental data have been reported, to our knowledgement. The transition probabilities supplied by Ali \& Kim (1988) comprise the ions up to Mo XXIV. These authors have used the multiconfiguration DiracFock (MCDF) code of Declaux (1975) in optimized level mode, which includes QED corrections. Another interesting set of comparing data are the $\mathrm{A}$ values reported by 
Table 5. E2 and M1 transition probabilities for Z-high ions.

\begin{tabular}{llllll}
\hline \hline ION & $Z$ & $\mathrm{E} 23 \mathrm{~d}_{3 / 2} \leftarrow 4 \mathrm{~s}_{1 / 2}$ & $\mathrm{E} 23 \mathrm{~d}_{5 / 2} \leftarrow 4 \mathrm{~s}_{1 / 2}$ & $\mathrm{E} 23 \mathrm{~d}_{3 / 2} \leftarrow 3 \mathrm{~d}_{5 / 2}$ & ${\mathrm{M} 13 \mathrm{~d}_{3 / 2} \leftarrow 3 \mathrm{~d}_{5 / 2}}$ \\
\hline Pr XLI & 59 & $3.21(+9)$ & $4.60(+9)$ & $3.97(+0)$ & $8.13(+4)$ \\
Nd XLII & 60 & $3.66(+9)$ & $5.25(+9)$ & $5.29(+0)$ & $1.02(+5)$ \\
Pm XLIII & 61 & $4.18(+9)$ & $5.98(+9)$ & $7.00(+0)$ & $1.27(+5)$ \\
Sm XLIV & 62 & $4.75(+9)$ & $6.78(+9)$ & $9.20(+0)$ & $1.58(+5)$ \\
Eu XLV & 63 & $5.38(+9)$ & $7.67(+9)$ & $1.20(+1)$ & $1.94(+5)$ \\
Gd XLVI & 64 & $6.08(+9)$ & $8.66(+9)$ & $1.56(+1)$ & $2.37(+5)$ \\
Tb XLVII & 65 & $6.85(+9)$ & $9.75(+9)$ & $2.02(+1)$ & $2.92(+5)$ \\
Dy XLVIII & 66 & $7.70(+9)$ & $1.09(+10)$ & $2.59(+1)$ & $3.55(+5)$ \\
Ho XLIX & 67 & $8.64(+9)$ & $1.23(+10)$ & $3.30(+1)$ & $4.29(+5)$ \\
Er L & 68 & $9.67(+9)$ & $1.37(+10)$ & $4.20(+1)$ & $5.19(+5)$ \\
Tm LI & 69 & $1.08(+10)$ & $1.53(+10)$ & $5.30(+1)$ & $6.21(+5)$ \\
Yb LII & 70 & $1.20(+10)$ & $1.70(+10)$ & $6.67(+1)$ & $7.43(+5)$ \\
Lu LIII & 71 & $1.34(+10)$ & $1.88(+10)$ & $8.36(+1)$ & $1.06(+5)$ \\
Hf LIV & 72 & $1.49(+10)$ & $2.09(+10)$ & $1.04(+2)$ & $1.25(+6)$ \\
Ta LV & 73 & $1.65(+10)$ & $2.31(+10)$ & $1.30(+2)$ & $1.48(+6)$ \\
W LVI & 74 & $1.82(+10)$ & $2.55(+10)$ & $1.60(+2)$ & $1.76(+6)$ \\
Re LVII & 75 & $2.01(+10)$ & $2.81(+10)$ & $1.97(+2)$ & $2.05(+6)$ \\
Os LVIII & 76 & $2.21(+10)$ & $3.09(+10)$ & $2.42(+2)$ & $2.40(+6)$ \\
Ir LIX & 77 & $2.44(+10)$ & $3.39(+10)$ & $2.96(+2)$ & $3.83(+6)$ \\
Pt LX & 78 & $2.67(+10)$ & $3.71(+10)$ & $3.61(+2)$ & $4.38(+2)$ \\
Au LXI & 79 & $2.93(+10)$ & $4.06(+10)$ & $5.30(+2)$ & \\
Hg LXII & 80 & $3.21(+10)$ & $4.43(+10)$ & & \\
\hline
\end{tabular}

Biémont (1990) for the ions Cd XXX-Ce XL, calculated, through a multiconfigurational Dirac-Fock approach, with Desclaux's (1975) code. However, in Biémont's calculations, the configuration interaction was retained including several configurations, some of which are of the $3 \mathrm{p}^{5} n l n^{\prime} l^{\prime}$ type. In these calculations, Breit interaction and QED corrections were accounted for. We consider the data supplied by Biémont (1990) to be highly reliable.

Results for E2 and M1 intraconfiguration transitions have also been reported by Biémont \& Hansen (1989). These authors followed a Relativistic Hartree-Fock approach (HFR) to built approximate relativistic HartreeFock wavefunctions with Cowan's code (1981). No other data, to our knowledge, are available in the literature.

An overall inspection of Tables 1-4 reveals that our RQDO $A$-values show in general good accord with the data reported by other authors.

The RQDO A-values for the E2 fine-structure transitions $3 \mathrm{~d}_{3 / 2} \leftarrow 4 \mathrm{~s}_{1 / 2}$ and $3 \mathrm{~d}_{5 / 2} \leftarrow 4 \mathrm{~s}_{1 / 2}$, displayed in Tables 1 and 2, respectively, are slightly lower in magnitude than the comparative data from $Z=30$ onwards. We attribute these discrepancies to the explicit account of configuration interaction in Biémont's calculations (1990) unlike in the present ones, where these effects are only implicitly included. However, the lack of convergence problems in the RQDO procedure is, in our view a great advantage when mass production of data is needed.
It is interesting to notice that for a small group of ions $(Z=43-47)$ there are no comparative data reported.

In Table 3, which displays data for the intraconfiguration E2 transition, $3 \mathrm{~d}_{3 / 2} \leftarrow 3 \mathrm{~d}_{5 / 2}$, a similar general behaviour in our data with respect to the comparative $A$ values is found. The RQDO data, except for the six first ions, are again smaller than the others also included in the table. Notice, however, the excellent agreement observed for the astrophysically important ion Fe VIII.

In Table 4 , the $A$-values for the M1 $3 \mathrm{~d}_{3 / 2} \leftarrow 3 \mathrm{~d}_{5 / 2}$ line are displayed. As in the previous fine-structure transitions, the overall agreement between the RQDO transition probabilities and the data reported by other authors is fairly good. The most extensive data for this transition are those reported by Biémont \& Hansen (1989), with which the agreement of the RQDO results is slightly better than with those by Ali \& Kim (1988).

Finally, in Table 5, we collect the RQDO A-values of the different $\mathrm{E} 2$ and M1 transitions for ions with $Z \geq 59$, for which no comparative data have been found.

The regularities of the intensity values along an isoelectronic sequence are of great practical importance, since they may be exploited to obtain additional oscillator strengths or transition probabilities by interpolation or extrapolation, as well as to evaluate the reliability of existing data by the degree of fit into established systematic trends (Wiese \& Weiss 1968). We have analysed the behaviour of the fine-structure transition probabilities along 
the potasium isoelectronic sequence as a function of the nuclear charge $Z$, and fitted the $A$-values for the entire group of ions for each transition to a polynomic function of $Z$. The best fitting formulae for the $\mathrm{E} 2$ emission lines, (a) $4 \mathrm{~s}_{1 / 2} \rightarrow 3 \mathrm{~d}_{3 / 2}$ and (b) $4 \mathrm{~s}_{1 / 2} \rightarrow 3 \mathrm{~d}_{5 / 2}$, are, respectively

$$
\begin{aligned}
A(\mathrm{a})= & -0.1083 Z^{6}+106.07 Z^{5}-14562 Z^{4}+904880 Z^{3} \\
& -3 \times 10^{7} Z^{2}+5 \times 10^{8} Z-4 \times 10^{9} \\
A(\mathrm{~b})= & -0.8176 Z^{6}+324.62 Z^{5}-39513 Z^{4}+210^{6} Z^{3} \\
& -8 \times 10^{7} Z^{2}+1 \times 10^{8} Z-9 \times 10^{9} .
\end{aligned}
$$

And for the intraconfiguration decay, $3 \mathrm{~d}_{5 / 2} \rightarrow 3 \mathrm{~d}_{3 / 2}$, via E2 and M1 mechanism, we have obtained, respectively,

$$
\begin{gathered}
A(\mathrm{E} 2)=4 \times 10^{-7} Z^{6}-10 \times 10^{-5} Z^{5}+0.0128 Z^{4} \\
-0.7566 Z^{3}+24.656 Z^{2}-419.32 Z+2903.2
\end{gathered}
$$

$$
\begin{aligned}
A(M 1)= & 0.0015 Z^{6}-0.3835 Z^{5}+41.907 Z^{4}-2419.6 Z^{3} \\
& +77494 Z^{2}-1 \times 10^{6} Z+9.10^{6}
\end{aligned}
$$

From these expressions, estimates for ions of lower or higher $Z$ than the ones presently studied may be obtained.

\section{Conclusions}

The RQDO procedure has once more proved to be a very useful tool for estimating transition probabilities. In the particular case of the K-like ions, a general, satisfying, agreement is found between the RQDO results and those obtained with rather more elaborate procedures.

Additionally, we have found that the RQDO $A$-values of the E2 and M1 fine structure lines follow systematic trends along the isoelectronic sequence, which have long been considered as a qualitative proof of correctness and can be exploited for the interpolation or extrapolation of non-calculated data. We are confident in that the presently supplied RQDO $A$-values for heavy ions of the potasium sequence may be potentially useful in astrophysics and fusion plasma research.

Acknowledgements. This work has been supported by the DGES of the Spanish Ministry of Education within Project No. PB97-0399-C03-01.

\section{References}

Adelman, S. J., \& Gulliver, A. F. 1999, Phys. Scr., T83, 97 Ali, M. A., \& Kim, Y.-K. 1988 Phys. Rev. A, 38 (8), 3992

Biémont, E. 1990 Bulletin de la Société Royale des Sciences de Liège, 59e année, 3-4, 319
Biémont, E., \& Hansen, J. E. 1989, Phys. Scr., 39(3), 308

Calamai, A. G., Gwinner, G., Tordoir, X., Trabert, E., \& Wolf, A. 2000, Phys. Rev. A, 6162508

Charro, E., \& Martín, I. 1998, A\&AS, 131, 523

Charro, E., Martín, I., \& Lavín, C. 1997, A\&AS, 124, 397

Charro, E., Martín, I., \& Serna, M. A. 2000, J. Phys. B., At. Mol. Opt. Phys., 33, 1753

Cowan, R. D. 1981, The Theory of Atomic Structure and Spectra (Univ. of California Press)

Declaux, J. P. 1975, Comput. Phys. Commun., 9, 31

Edlén, B. 1984, Phys. Scr., T85(3)

Fournier, K. B., Goldstein, W. H., May, M., \& Finkenthal, M. 1996, Phys. Rev. A, 53(2), 709

Gabriel, A. H., \& Jordan, C. 1969, MNRAS, 145, 241

Hinnov, E., \& Suckewer, S. 1980, Phys. Lett. A, 32, 298

Karwowski, J., \& Martín, I. 1991, Phys. Rev. A, 43, 4832

Kaufman, V., Sugar, J., \& Rowan, W. L. 1989, J. Opt. Soc. B, 6(3), 142

Kelly, R. L. 1987, J. Chem. Ref. Data, 16, Suppl. 1

Martín, I., Almaraz, M. A., \& Lavín, C. 1995, Z. Phys. D, 35, 228 and references therein

Martín, I., Lavín, C., \& Charro, E. 2000a, in New Trends in Quantum Systems in Chemistry and Physics, vol. 2, ed. J. Maruani, et al. (Kluwer Academic Publishers, Dordrecht), 49

Martín, I., \& Karwowski, J. 1991, J. Phys. B, At. Mol. Opt. Phys., 24, 1538

Martín, I., Karwowski, J., \& Bielinska-Waz 2000b, J. Phys. A, Math. Gen., 33, 823

Martín, I., Karwowski, J., Lavín, C., \& Diercksen, G. H. F. 1991, Phys. Scr., 44, 567

Martín, P, Lavín, C., \& Martín, I. 1994, Z. Physik. D, Atoms, Molecules and Clusters, 30, 279

Martin, W. C., \& Wiese, W. L. 1996, in Atomic, Molecular, and Optical Physics Handbook, ed. G. W. F. Drake (American Institute of Physics, Woodbury, New York), 135

Osterbrock, D. E. 1974, Astrophysics of Gaseous Nebulae (Freeman, San Francisco)

Osterbrok, D. E. 1989, Astrophysics of Gaseous Nebulae and Active Galactic Nuclei (University Science Books, Mill Valley, CA)

Seaton, M. J. 1968, Adv. Atom. Molec. Phys., 4, 331

Sobelman, I. I. 1979, Atomic Spectra and Radiative Transitions (Springer-Verlag)

Suckewer, S., Fonck, R., \& Hinnov, E. 1980, Phys. Rev. A, 21, 929

Suckewer, S., \& Hinnov, E. 1979, Phys. Rev. A, 20, 578

Sugar, J., \& Corliss, C. 1985, J. Phys. Chem. Ref. Data, 14, Suppl. 2, 650

Wang, M., O'Reilly, F., Dunne, P., et al. 1997, J. Phys. B., At. Mol. Opt. Phys., 30, 4175

Wang, M., Faulkner, R., O'Reilly, F., et al. 1999, J. Phys. B., At. Mol. Opt. Phys., 32, 5299

Wiese, W. L., \& Weiss, A. W. 1968, Phys. Rev., 175(1), 50 